\title{
Tic symptoms in Tourette's syndrome reached their worst level of severity in middle childhood and then declined
}

Leckman JF, Zhang H, Vitale A, et al. Course of tic severity in Tourette syndrome: the first two decades. Pediatrics 1998 Jul;102:14-9.

\section{Questions}

In patients with Tourette's syndrome (TS), what is the course of tic symptoms over the first 20 years of life? Is onset of puberty associated with the timing or level of worst ever tic severity?

\section{Design}

Inception cohort followed up for a mean of 7.5 years (range 1 to 12 y).

\section{Setting}

A specialty tic disorders clinic at a children's mental health centre in the US.

\section{Patients}

36 patients $(89 \%$ boys, mean age 11 y) born in 1975 who had been diagnosed with TS (according to DSM-III or DSM-III-R criteria) and evaluated at the Yale Child Study Center Tic Disorders Clinic and who had participated in a case control study on the time course of tic severity.

\section{Assessment of prognostic factors}

A chart review and 5 interviews ( 1 telephone and 4 personal) with parents were used to obtain data on demographics; presence, severity, and age at onset of TS, attention deficit hyperactivity disorder, and obsessive compulsive disorder; other comorbid conditions; onset and duration of puberty; and parental tic history and other psychopathology.

\section{Main outcome measure}

Current and worst ever tic severity were assessed using the Yale Global Tic Severity Scale (YGTSS) (range 0 to 50 points) in an interview with the patient and parents.

\begin{abstract}
Main results
The mean age of tic onset was 5.8 years, and the mean age at which symptoms were at their worst was 10 years (range 6 to 15 y). The mean worst ever tic severity score on the YGTSS was 29.8. During the period of worst ever tic severity, $22 \%$ of patients had severe tics (YGTSS scores 40 to $<50$ ), 28\% had marked tic severity (YGTSS scores 30 to <40), 39\% had moderate tic severity (YGTSS scores 20 to $<30$ ), and 4\% had mild tic severity (YGTSS scores <20). Greater severity of tic symptoms during the worst ever period was associated with increasing age at the time of worst ever symptoms $(r=0.58, \mathrm{p}<0.001)$. By 18 years of age, $47 \%$ of patients had no tics, $11 \%$ had minimal tic symptoms (YGTSS score < 10), 28\% had mild symptoms (YGTSS score 10 to $<20$ ), and $11 \%$ had moderate or marked tic severity (YGTSS scores 20 to $<40$ ). Tic severity at baseline was not associated with worst ever or current tic severity. Tic severity at follow up was associated with tic severity during the worst ever period $(r=0.37, \mathrm{p}<0.03)$. The onset of puberty was not associated with the timing or degree of worst ever tic severity.
\end{abstract}

\section{Conclusions}

In patients with Tourette's syndrome, tics began at a mean age of 5.8 years and were most severe at a mean age of 10 years. The level of severity of tic symptoms during the worst ever period was associated with older age during this period but not at baseline. By 18 years of age, most patients were free of symptoms; tic severity at follow up was associated with tic severity during the worst ever period. The onset of puberty was not associated with the timing or degree of worst ever tic severity.

Sources of funding: March of Dimes and National Institutes of Health.

For correspondence: Dr J F Leckman, Room I-269 SHM, Child Study Center, Yale University School of Medicine, PO Box 207900, New Haven, CT 06520-7900, USA. Fax +1 2037857611.

\section{Commentary}

Leckman et al describe the course of tic severity in a birth cohort of patients with TS. The authors have minimised the limitations of a retrospective study, except for the fact that the time course of tic severity can fluctuate on a scale of weeks to months. This was not captured in the study because the time scale of years was used.

Two previous studies based on physician surveys in South Dakota showed a lower prevalence of TS in adolescents than in pre-adolescents. A study that used direct observation of unselected schoolchildren 13-14 years of age, however, found a prevalence of $3 \%$, which is higher than previous estimates based on less sensitive methodology. ${ }^{1}$ This supports Leckman et al's suggestion that TS is a common but relatively mild and self limiting condition which does not necessarily cause substantial distress or interference after 18 years of age. No data, however, are presented to show that "starting medications may do more harm than good." Certainly, medication should only be offered when symptoms are sufficiently severe to disrupt psychosocial development, educational progress, or family dynamics. To not offer treatment in such situations may have adverse effects, and the issue of whom to treat requires further study.

Finally, it is worth noting that although tics in early adulthood may decline to undetectable levels, the diagnosis of TS still applies. Ample evidence shows that TS is a lifelong, genetic, neuropsychiatric disorder of childhood onset with probable genetic aetiology. ${ }^{2}$ Hence, children whose parents have mild symptoms or a remote history of
TS in childhood or adolescence are still at increased risk of developing TS compared with the general population. This recalls the current controversy about diagnostic criteria for TS in DSM-III-R as opposed to $D S M-I V .{ }^{3}{ }^{4}$ The diagnostic criteria in DSM$I V$ require distress and interference to make the diagnosis. These strictures cannot be used in genetic research and would be misleading when a patient requests genetic counselling.

Paul Sandor, MD

The Toronto Hospital, Western Division Toronto, Ontario, Canada

1 Mason A, Baneriee S, Eapen V, et al. Dev Med Child Neurol 1998;40:292-6.

2 Pauls DL, Raymond CL, Stevenson JM, et al. Am

JHum Genet 1991;48:154-63.
Kurlan R. Arch Neurol 1997;54:517-8.

4 Freeman RD, Fast DK, Kent M. JAm Acad Child Freeman RD, Fast DK, Kent M.J Anc Asychiatry 1995;34:400-1.
Adolesc 